\title{
Far-infrared and sub-millimeter properties of SDSS galaxies in the Herschel ATLAS SDP Field
}

\author{
Man I. Lam ${ }^{1,2}$, Hong $\mathbf{W u}^{1}$ and $\mathrm{Yi}$-Nan $\mathbf{Z h u}^{1}$ \\ ${ }^{1}$ National Astronomical Observatories, CAS Beijing, 100012, P. R. China \\ ${ }^{2}$ Graduate University of the Chinese Academy of Sciences, Beijing, 100049, P. R. China \\ email: hwu@bao.ac.cn
}

\begin{abstract}
Using data from the new infrared facility the Herschel Space Observatory, we have analyzed correlations between morphological type, far-infrared (FIR) luminosity, and H $\alpha$ luminosity for star-forming galaxies, composite galaxies, and AGNs. We found a trend in scatter from $100 \mu \mathrm{m}$ to $500 \mu \mathrm{m}$, which indicates that the submillimeter bands are not a good star formation tracer in these galaxies, being contaminated either by the old stellar population or by the interstellar medium (ISM). AGNs have no significant effect on our fitting results since the far-infrared to submillimeter emission is from cold dust/large dust grains.
\end{abstract}

Keywords. galaxies: starburst, galaxies: ISM, galaxies:classification, infrared: galaxies

\section{Introduction}

Infrared astronomy developed spectacularly since IRAS was launched in 1983, and discovered a type of peculiar object called ultra luminous infrared galaxies (ULIRGs) (Sanders \& Mirabel 1996). More space-based infrared telescopes have helped us to study the MIR to FIR emission properties of galaxies from that time, such as ISO, and Spitzer (Lonsdale et al. 2006, Soifer et al. 2008). Herschel is the first FIR space telescope which can extend the observation from FIR to the submm range to reveal the cold dust properties in our universe.

The MIR and FIR emission of galaxies are usually an indirect detection of extragalactic star formation activities, since infrared emission originates from the dust re-radiation of UV photons emitted by young stellar population. Most of the stellar light is emitted in the UV to near-IR domain, with the short-lived, massive stars dominating the UV and the more numerous older stars the near-IR. Dust, produced by the aggregations of metals injected into the ISM by massive stars through stellar winds and supernovae, absorbs the stellar light and reemits it in the IR and submm domains. At shorter FIR wavelengths (e.g. less than $200 \mu \mathrm{m}$ ) luminosity has been proved to be a good star formation tracer of galaxies by IRAS observations. Also, previous studies in the MIR using Spitzer observations indicate that the MIR emission is well correlated with the $1.4 \mathrm{GHz}$ and $\mathrm{H} \alpha$ luminosities. The longer wavelength properties of galaxies are however still unclear, especially in the bands which are dominated by the continuum from big grains. Some clues offered by nearby galaxies show that the older stellar population can also heat dust grains, and dominate the far-infrared luminosity. 

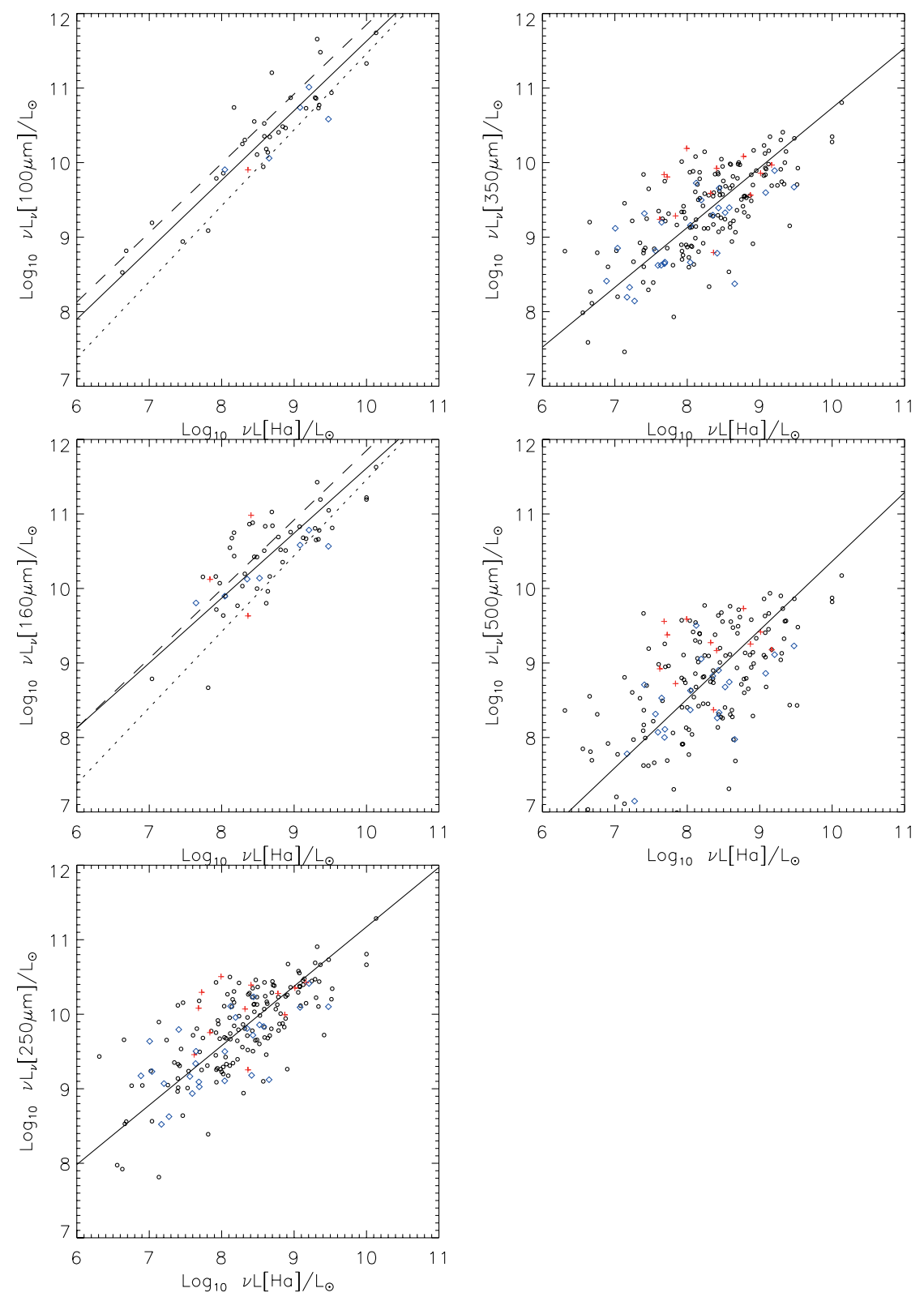

Figure 1. The L(FIR)-L(H $\alpha)$ correlation. Circles: Spiral galaxies. Crosses: E/S0 galaxies (which show an obvious bias with respect to the fitted line. Diamonds: AGN-dominated galaxies.

\section{Sample}

We used the first release of data from the Herschel ATLAS survey. In order to compare the IR/submillimeter properties of galaxies with different galamorphological types, only sample galaxies with SDSS optical spectra and redshift $z<0.3$ were selected in our final sample. This sample included 297 objects without obvious lensing.

We divided our sample galaxies into six different morpholocial groups based on de Vaucouleurs et al. (1991), which included E/S0, early than Sb, later than Sb, Peculiar, and 
Compact. The peculiar type represented the galaxies with peculiar morphologies, either irregular galxies or asymmetric morphologies. The sample is thereby divided into fractions E/S0:S(Sa-Sd):Pec:Compact $=0.08: 0.38: 0.34: 0.08$. Obviously, our sample is dominated by later type galaxies.

\section{Results}

L/M Ratio in different Hubble Types The $\mathrm{L} / \mathrm{M}$ ratio is influenced by the fraction of gas to stars. In our sample, L(FIR)/M with different Hubble types show a shift between different FIR/Submm bands. The average L/M ratios become higher with later Hubble types. The peculiar galaxies have markedly higher $\mathrm{L}(\mathrm{FIR}) / \mathrm{M}$ ratio than the other five types, because these galaxies have larger gas reservoirs. Also, the peculiar galaxies have higher $\mathrm{L}(\mathrm{H} \alpha) / \mathrm{M}$ ratios, since these galaxies have a larger fraction of young stars.

$L(F I R)-L(H \alpha)$ relations Far-infrared and $\mathrm{H} \alpha$ emissions are usually used as starformation tracers. We present in Fig. 1 the correlations between 'total' FIR and 'total' $\mathrm{H} \alpha$ luminosity after K-correction, dust extinction correction and aperture correction. The $\mathrm{L}(\mathrm{FIR})-\mathrm{L}(\mathrm{H} \alpha)$ relation shows a trend with Spearman rank coefficient from 0.89 on $100 \mu \mathrm{m}$ to 0.56 on $500 \mu \mathrm{m}$. The equivalent width has an obvious influence on the fitting. Galaxies with lower equivalent widths deviate from the the best fitting line in our sample. AGNs do not significantly affect the FIR/submm luminosites, since the SEDs of AGNs are power laws from the UV to the infrared, so that the far-infrared amplitude is small.

\section{Discussion}

$L(F I R)-L(H \alpha)$ Scatter The trend of L(FIR)-L(H $\alpha)$ correlation seems to show that the flux in FIR are not just from star formation. Therefore, FIR/Submm emission may be not a good star formation tracer. The FIR emission is more complicated than our previous assumption.

FIR from Early Type Galaxies Some early type galaxies with a very red colour were found in our sample. They are lacking in $\mathrm{H} \alpha$ emission, instead exhibiting stronger $\mathrm{H} \beta$ and sodium absorption lines. These galaxies indicate that we cannot ignore the contribution from old stellar populations in the far-infrared. Besides, we also found some sources with weak $\mathrm{H} \alpha$ line emission and a strong continuum from older stellar populations. These sources may be either post-starburst galaxies or undergoing a minor merger event. Although still involved in a starburst, a smaller fraction of the far-infrared luminosity is contributed by young stars in these galaxies.

\section{Acknowledgment}

We gratefully thank Hershel ATLAS group, SDSS, and the SED2011 conference organizer.

\section{References}

Sanders, D. B. \& Mirabel, I. F. 1996, ARAA, 34, 749S

Lonsdale, C. J., Farrah, D., \& Smith, H. E. 2006, asup.book, 285L

Soifer, B. T., Helou, G., \& Werner, M 2008, ARAA, 46, 201S

de Vaucouleurs, G., de Vaucouleurs, A., Corwin, H. G., Buta, R. J., Paturel, G., \& Fouqué, P., Third Reference Catalogue of Bright Galaxies, 1991, Springer 